\title{
An Adiabatic Multiple Spin-Echo Pulse Sequence: Removal of Systematic Errors Due to Pulse Imperfections and Off-Resonance Effects
}

\author{
Markus Zweckstetter and Tad A. Holak \\ Max Planck Institute for Biochemistry, D-82152 Martinsried bei München, Germany
}

Received August 12, 1997; revised February 27, 1998

\begin{abstract}
A pplication of AFP (adiabatic fast passage) pulses for removal of systematic errors associated with multiple spin- echo sequences is demonstrated. The adiabatic fast passage pulses facilitate minimization of cumulative pulse errors for all three components of magnetization. It is also shown that off-resonance effects present in conventional CPMG sequences which degrade image quality in magnetic resonance imaging and introduce systematic errors in measured $T_{2}$ relaxation time peak amplitudes can be suppressed by introduction of AFP pulses without any degradation of overall signal intensity. The technique has been tested on the ${ }^{15} \mathrm{~N}$ spinspin relaxation time measurements of a 110 amino acid domain of the F-actin cross-linking protein. $\odot 1998$ Academic Press
\end{abstract}

Key Words: spin- echo; adiabatic fast passage pulses; AFPCPMG ; AFP-MLEV-4; $T_{2}$ relaxation time.

\section{INTRODUCTION}

Multiple spin-echo (MSE) sequences $(1,2)$ are widely used in magnetic resonance imaging (MRI) of tissues $(3,4)$ and in nuclear magnetic resonance (NMR) relaxation studies of biomolecules (5). Both applications are based on the formation of echoes decaying exponentially with the transverse spin-spin relaxation time $T_{2}$ in the absence of imperfections. MSE sequences, originally introduced by Carr and Purcell to minimize the influence of molecular diffusion (1), suffer from cumulative effects of imperfect $180^{\circ}$ pulses. Deviations from a pure $180^{\circ}$ rotation of a single refocusing pulse can originate from mistuned pulse parameters, from static field inhomogeneities, from spatial inhomogeneity of the radiofrequency (RF) field intensity over the sample volume, and from off-resonance effects. An imperfect refocusing pulse employed in a MSE sequence then gives rise to unwanted echoes $(6-11)$, phase distortions, and off-resonance dependent intensity losses of the signal of interest $(12,13)$. These deleterious effects result in image artefacts in MRI and in a decay of the magnetization which is not solely governed by irreversible spin-spin relaxation processes $(6-13)$. The latter effect leads to substantial systematic errors in $T_{2}$ measurements and therefore to degradation in MRI image quality $(6-12)$ or misinterpretation of protein mobility by NMR (13). To overcome these problems a variety of approaches have been suggested. Meiboom and Gill showed that application of a $90^{\circ}$ phase shift between the $90^{\circ}$ excitation and $180^{\circ}$ refocusing pulses can be used to preserve the magnetization which is in-phase with the refocusing pulses despite pulse imperfections (2). Phase alternating schemes have been suggested for experiments in which it is essential to remove cumulative errors for all three magnetization components $(14-16)$. Other proposals were the use of gradients to defocus unwanted echoes $(6-8)$ and suppress off-resonance effects (17). The use of composite pulses (18) for elimination of systematic errors was also investigated but only with limited success $(7,12,13)$. All these methods suffer, however, from some kind of drawbacks. On the other hand, pulses based on adiabatic fast passage (AFP) $(19,20)$ can achieve inversion of magnetization in the presence of large frequency offsets and with high tolerance to spatial variations in the RF field intensity $(21,22)$. A spectral phase roll is observed as a function of frequency offset after a single AFP pulse is used as a refocusing element (23). Elimination of this phase roll can be achieved by optimizing shapes of AFP pulses $(24,25)$, by composite adiabatic pulses $(23,26-28)$, by a series of AFP pulses separated by flips of the effective magnetic field $\mathbf{H}_{\text {eff }}$ (29), or by the formation of even-numbered echoes using any pair of identical adiabatic inversion pulses $(18,30)$.

In this contribution we demonstrate that two identical adiabatic fast passage pulses used as a double refocusing element in MSE sequences remove systematic errors in $T_{2}$ measurements. Deleterious effects due to both pulse imperfections and off-resonance effects are suppressed. The method is insensitive to radiofrequency field inhomogeneity, works well for very large sweep widths, and, utilizing a MLEV-4 cycling scheme (31), preserves all three components of magnetization. The MSE sequences augmented with AFP pulses were tested on the ${ }^{15} \mathrm{~N} T_{2}$ relaxation times of amide backbones of segment 4 of the rod domain of a F-actin cross-linking protein, the gelation factor from $D$. discoideum (32).

\section{THE ORY}

In order to better understand the importance of achieving the most perfect $180^{\circ}$ rotation in MSE sequences and to appreciate 
the usage of AFP pulses in MSE sequences, we briefly outline the theoretical foundations of AFP pulses and the cumulative pulse errors in MSE sequences.

According to the Bloch equations in a frame rotating with the frequency of the applied RF field $\omega_{\mathrm{rf}}$ a single pulse of duration $t_{\mathrm{p}}$ and RF field $\mathbf{H}_{1}$ rotates the macroscopic magnetization $\mathbf{M}$ about an effective field $\mathbf{H}_{\mathrm{eff}}(\mathbf{r})=\mathbf{H}_{1}(\mathbf{r})+\Delta \mathbf{H}$ (33, 34) by an angle

$$
\theta=\gamma t_{\mathrm{p}} H_{\mathrm{eff}} .
$$

Therein $\Delta \mathbf{H}$ represents the offset field caused either by different chemical environments experienced by the nuclear spins, $\Delta \mathbf{H}=\mathbf{H}_{0}+\omega_{\mathrm{rf}} / \gamma=-\omega_{\text {off }} / \gamma(\gamma$ : gyromagnetic ratio $)$, or by static magnetic field inhomogeneities. In the presence of the offset field the rotation therefore takes place about the effective field $\mathbf{H}_{\text {eff }}$, which makes an angle $\phi=\arctan \left(\Delta H / H_{1}\right)$ with $\mathbf{H}_{1}$. From these equations it can be seen that different sources can give rise to a deviation of $\theta$ from the desired $180^{\circ}$. These sources are either incorrectly set $t_{\mathrm{p}}$, a spatially varying or mistuned RF field intensity $H_{1}$, or an offset field $\Delta \mathbf{H}$. For simple pulses none of these imperfections can be removed completely; both incomplete phase reversal of the transverse component takes place, i.e., antiphase magnetization is generated, and a longitudinal magnetization component is created. Employing such pulses in a MSE sequence leads to a complex interaction of different magnetization components as antiphase components can be rephased and longitudinal components rotated back into the transverse plane. As a result the magnetization in a MSE sequence with $n$ refocusing pulses no longer decays exponentially with the transverse spin-spin relaxation time $T_{2}, M_{n}=M_{0} \exp \left(-2 n \tau / T_{2}\right)$, where $\mathbf{M}_{0}$ is the magnetization after a perfect $90^{\circ}$ excitation pulse, but is given by the relation

$$
M_{n}=M_{0} f_{n}\left(\theta, \phi, \tau, T_{1}, T_{2}\right) .
$$

$f_{n}\left(\theta, \phi, \tau, T_{1}, T_{2}\right)$ depends nonlinearly on different parameters: it oscillates as a function of the effective flip angle $\theta(7-13)$, the frequency offset $\omega_{\text {off }}(12,13)$, and the echo delay $\tau(13)$. It is also a function of the spin-lattice relaxation time $T_{1}$ due to contributions to the signal from components that spent part of the pulse sequence along the static magnetic field $(8-10)$.

For pulses based on adiabatic fast passage principles $\mathbf{H}_{1}$ is a function of time $t$ with $t \epsilon\left[0, t_{\mathrm{p}}\right]$. In the case of the well-known hyperbolic secant (HS) pulse (22), for example, the frequency is modulated according to $\Delta \omega(\max ) \tanh \left(\beta \tau^{\prime}\right)$ with $\tau^{\prime}=1-$ $2 t / t_{\mathrm{p}}, \Delta \omega(\max )$ is the maximum frequency excursion, and the truncation factor $\beta$ is chosen $\operatorname{such}$ that $\operatorname{sech}(\beta)=0.01$. The HS pulse has the advantage of an offset-independent adiabacity, i.e., $d / d t\left[H_{1}(t)\right] \ll \gamma H_{1}^{2}(t)$, for the whole duration of the pulse. Therefore, provided that the RF field intensity $H_{1}$ exceeds a certain threshold, the angle between the magnetization and the direction of the effective magnetic field $\mathbf{H}_{\text {eff }}$ is a constant of the motion during the pulse. As a result, magnetization which is transverse before the pulse is also perfectly transverse at the end of the AFP pulse when $\mathbf{H}_{\text {eff }}$ is antiparallel to $\mathbf{H}_{0}$. Since $\mathbf{H}_{\text {eff }}$ depends on the frequency offset the spins experience a frequency-dependent phase variation $\psi$ during the application of the pulse given by

$$
\psi(t)=\gamma \int_{0}^{t} H_{\mathrm{eff}}\left(t^{\prime}\right) d t^{\prime}
$$

in addition to the phase reversal. However, if one applies a second identical AFP pulse to the system this offset-dependent phase roll does not add up but cancels because the frequency sweep is performed in the same direction. This is equivalent to an inversion of $\mathbf{H}_{\text {eff }}$ between the two AFP pulses (23). To demonstrate this phase compensation, the evolution of magnetization during a double spin-echo (DSPE) with two identical HS pulses for a system of two scalar coupled spins in an isotropic medium has been simulated (Fig. 1A). Thus, neither off-resonance effects nor pulse imperfections lead to deviations from a pure exponential decay of transverse magnetization with increasing echo number for even-numbered echoes in a MSE sequence employing AFP pulses.

\section{RESULTS AND DISCUSSION}

Three questions must be addressed in order to assess the possibility of employing adiabatic fast passage pulses in multiple spin-echo sequences. First, is the stability of the hardware sufficient to generate two equal AFP pulses so that the phase roll is compensated in a double spin-echo? Second, are systematic errors associated with standard MSE trains eliminated when replacing the rectangular hard power pulses by AFP pulses? Third, does the usage of AFP-MSE trains in standard experiments lead to error free results without any degradation in overall signal intensity compared to the case when a standard CPMG sequence is employed?

\section{Quality of an AFP Double Spin-Echo Sequence}

In Fig. 1A the simulated time evolution of in-phase single quantum coherence during a double spin-echo with two identical HS pulses for a system of two scalar coupled spins in an isotropic medium is shown. Both chemical shift evolution and divergence of spin vectors due to scalar coupling are refocused. The mechanism of the scalar coupling refocusing is similar to that of chemical shift refocusing. The experimentally recorded refocusing profiles of a single HS pulse and two sequentially applied identical HS pulses are presented in Fig. 1B. In the upper panel of Fig. 1B the offset-frequency-dependent, quadratic phase roll produced by a single HS pulse employed as a refocusing element is clearly visible. This nonlinear phase variation with respect to offset frequency would complicate the use of AFP pulses in $T_{2}$ relaxation time measurements. In the 
A

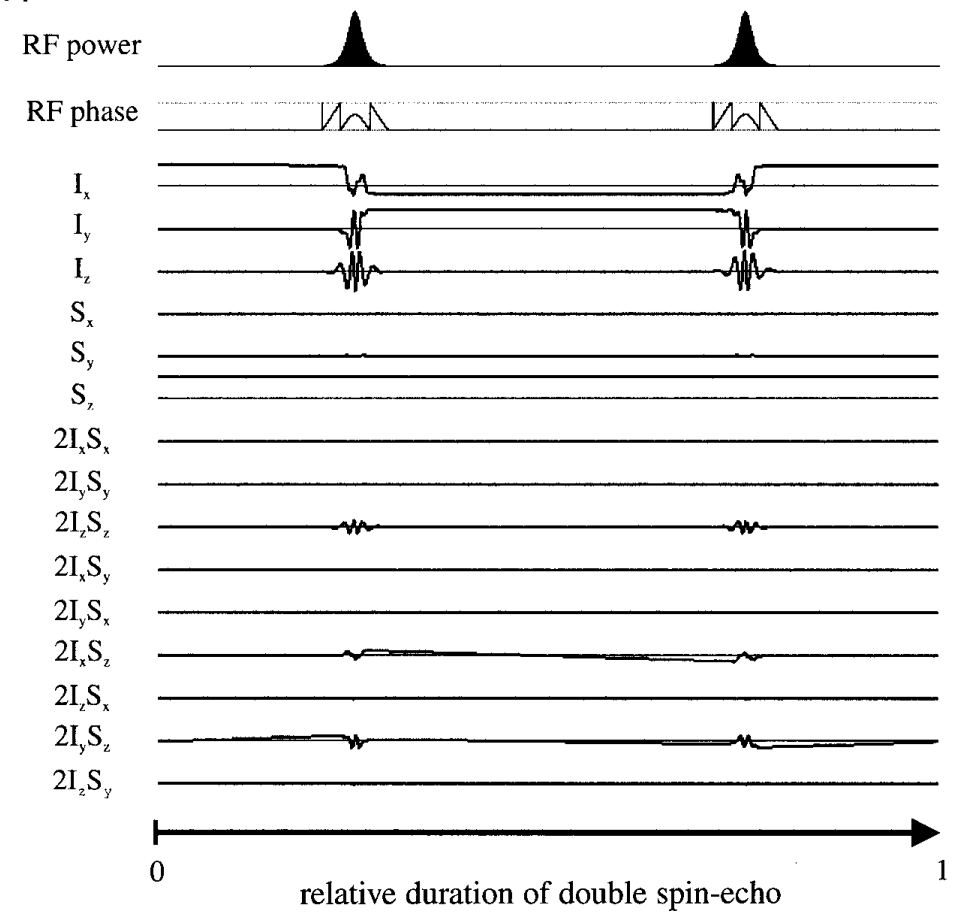

B
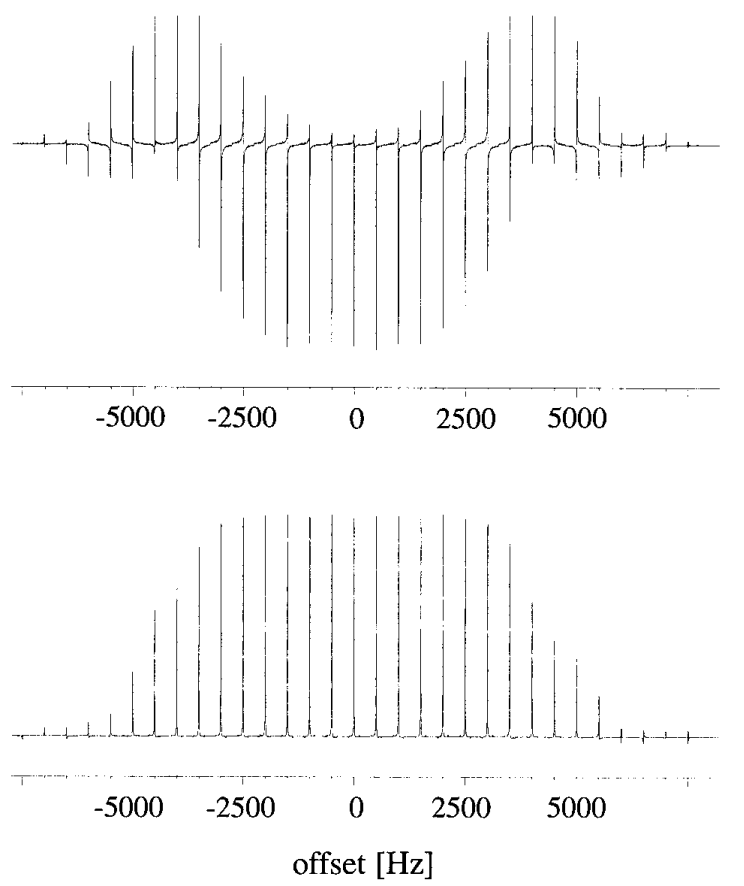

FIG. 1. (A) Simulation of the time evolution of a starting magnetization $I_{x}+S_{z}$ for a 2 -spin system in an isotropic medium during a double spin-echo experiment employing two HS pulses. The AFP pulses were on-resonance with respect to $I_{x}$, and had relative phase $x$, a duration of $t_{\mathrm{p}}=360 \mu \mathrm{s}$, a sweep width of $20 \mathrm{kHz}$, and a maximal RF field intensity of $\gamma \mathrm{H}_{1}^{\max }=22 \mathrm{kHz}$. The $S$ spin had a chemical shift offset of $550 \mathrm{MHz}$ with respect to the $I$ spin (chemical shift $=$ $0 \mathrm{~Hz}$ ) and was weakly coupled to the $I$ spin via a scalar coupling constant of $90 \mathrm{~Hz}$ (approximating the situation found for the backbone amide atom and the bound amide proton in proteins at $11.4 \mathrm{~T}$ ). The absolute duration of a single de/refocusing delay was $1 \mathrm{~ms}$. Relaxation effects were neglected. Time evolutions of all possible coherences in a system of two $\frac{1}{2}$ spins are shown. The simulation was performed with the program PENCIL (47) utilizing the Bloch equations (33). (B) Symmetric refocusing profiles of a single HS pulse (22) and two identical HS pulses (upper and lower panel, respectively). De/refocusing delays were 0.82 $\mathrm{ms}$. The resonance offset was stepped from -7.5 to $7.5 \mathrm{kHz}$ in increments of $500 \mathrm{~Hz}$. The on-resonance signal was phased into absorption mode and the same phase correction was applied to all other peaks. Prior to Fourier transformation an exponential window function of $0.3 \mathrm{~Hz}$ was applied.

lower panel of Fig. 1B the profile shown was obtained by using two identical HS pulses as a double refocusing element. As in the simulation in Fig. 1A phase distortions are completely removed. Moreover, there is no reduction in overall signal intensity with increasing frequency offset within a frequency range of at least $6 \mathrm{kHz}$, i.e., for at least $60 \%$ of the frequency sweep width of the HS pulse. The same perfect phase compensation was obtained for echo delays ranging from 0.32 to $10.32 \mathrm{~ms}$, indicating that the hardware is stable enough to generate two HS pulses which are sufficiently identical for our application.

Disadvantages of a double spin-echo with AFP pulses are an increased minimal total length of the spin-echo sequence due to the longer AFP pulses and that an echo with no phase distortion is obtained only for even-numbered echoes. However, increased minimal total time length of the echo sequence could be a problem, if at all, only in imaging, and a remedy for this has already been proposed (30). The number of pulses and the requirement for even-numbered echoes do not represent problems for the application investigated here. It is thus clear that with modern hardware two identical AFP pulses can be used as a double refocusing element without producing any phase distortion of the signal of interest. Only on older instru- ments might the stability of the hardware not be sufficient to achieve complete compensation of the phase roll. In addition, AFP pulses are insensitive to RF field inhomogeneity, and even extremely large frequency offsets are feasible as the sweep width of AFP pulses is not limited.

\section{Elimination of Systematic Errors Due to Pulse Imperfections}

Figure 2 shows the results of extending the double spinecho with AFP pulses into a MSE train. Spin-echo amplitudes for an increasing number of double spin-echoes were recorded on-resonance and at $2 \mathrm{kHz}$ off-resonance in order to investigate whether RF field inhomogeneity or pulse imperfections lead to reduction of signal intensity with increasing number of AFP pulses. To find out whether magnetization components parallel and perpendicular to the phase of the AFP pulses are treated equally, amplitude profiles of each MSE train were recorded twice with the overall phase of the MSE sequence differing by $90^{\circ}$. In Fig. 2A the deleterious effect of the imperfections of rectangular hard power pulses, when used in a $\mathrm{CP}$ train, are clearly visible. Deviations from a pure $180^{\circ}$ rotation accumulate and lead to rapid decay of the signal. When applying a $90^{\circ}$ phase shift to the refocusing pulses relative to the excitation 
A

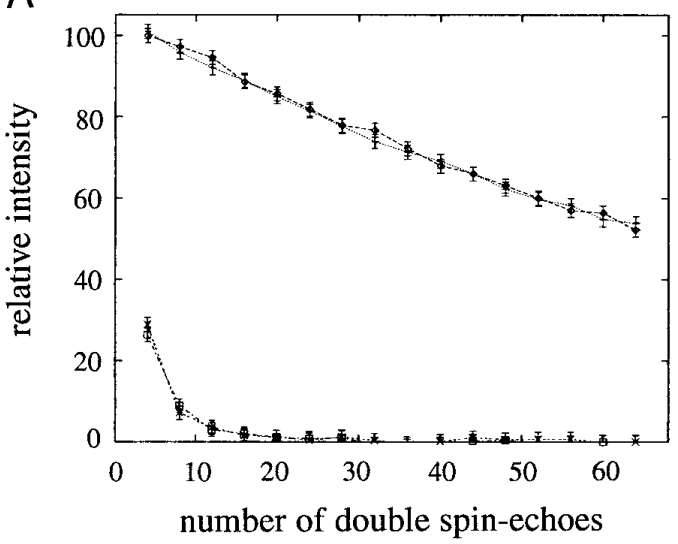

C

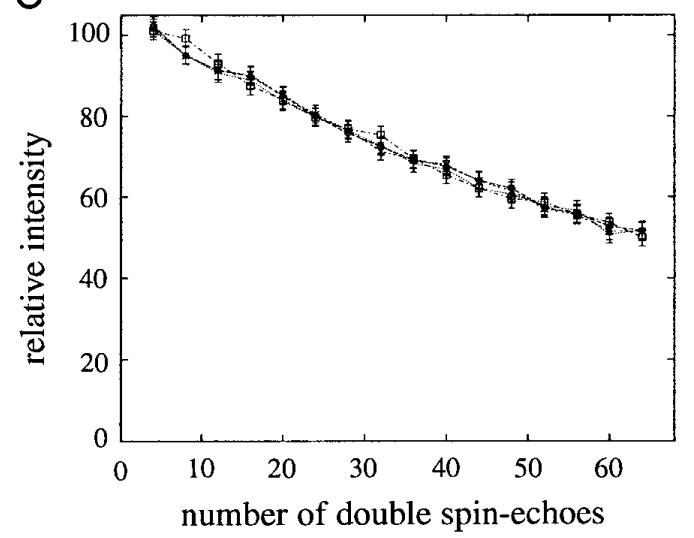

B

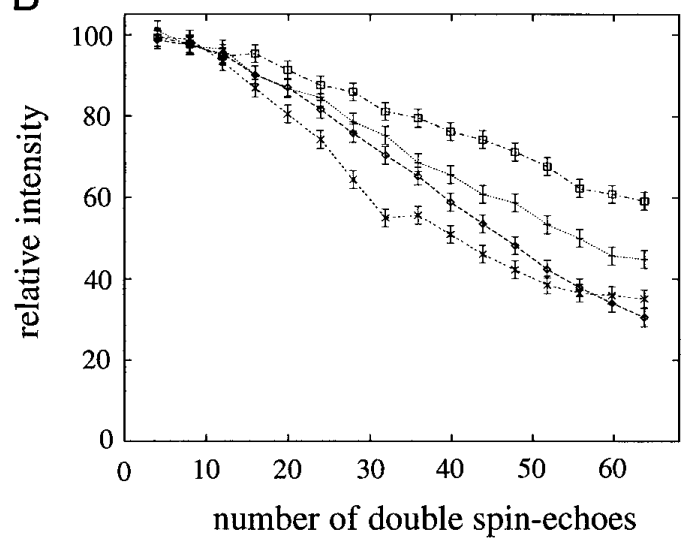

D

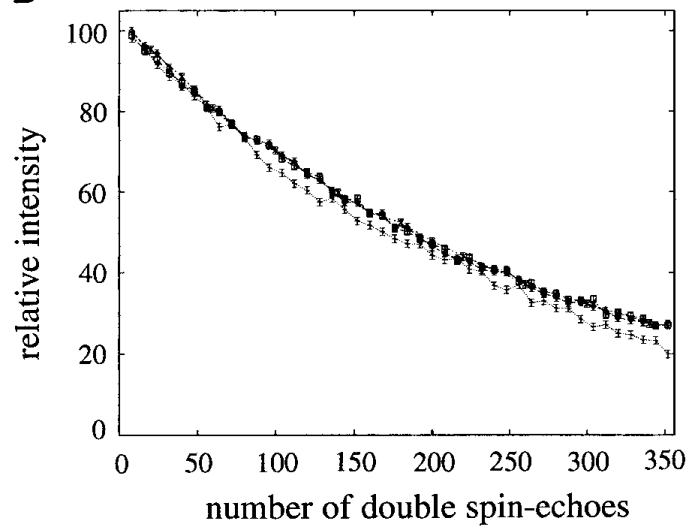

FIG. 2. Relative echo amplitudes for increasing number of double spin-echoes for various MSE trains on-resonance and at $2 \mathrm{kHz}$ off-resonance for both relative phase $y$ and relative phase $x$ of the MSE train. In all cases the recovery delay was set to $5 \mathrm{~s}$ and a rectangular hard power pulse (phase $x$ ) of RF field intensity $33.3 \mathrm{kHz}$ was used for excitation of transverse magnetization. For the amplitude profiles shown in (A) to (C) a single spin-echo had a duration of 3 $\mathrm{ms}$ and for those displayed in (D) of $1.1 \mathrm{~ms}$. The phase of the excitation pulse and the receiver were cycled along $x$ and $-x$ to select only magnetization originating from the first pulse. The experimental errors are discussed under Experimental. All echo amplitudes have been scaled relative to the spin-echo amplitude obtained with four double spin-echoes employing rectangular hard power pulses of phase $y$ for refocusing. In (A) to (C) the different experimental conditions are indicated in the following way: overall phase $y$ and on-resonance $(---/ \diamond)$; overall phase $y$ and $2 \mathrm{kHz}$ off-resonance $(\cdots / \dagger)$; overall phase $x$ and on-resonance $(-\cdot-\cdot$ $/ \square$ ); overall phase $x$ and $2 \mathrm{kHz}$ off-resonance (- - -/×). (A) Hard power rectangular refocusing pulses with $\gamma \mathrm{H}_{1}=33.3 \mathrm{kHz}$. CPMG, overall relative phase $y$ of MSE train; CP, overall relative phase $x$. (B) HS pulses $\left(t_{\mathrm{p}}=1 \mathrm{~ms}\right.$, sweep width $=10 \mathrm{kHz}, \gamma \mathrm{H}_{1}^{\max }=24.5 \mathrm{kHz}$, digitization $=1024$ points). (C) As (B) but incorporating the AFP-DSPEs into a MLEV-4 cycle. (D) Comparison of long-term behavior of various MSE sequences: CPMG (-- $-/ \triangleright$ ), AFP-MLEV-4- $y$ $(\cdots /+), X Y-16-x(-\cdot-\cdot / \square)$, AFP-MPFn- $x(---/ \times)$ (note the large number of DSPEs!).

pulse, i.e., using a CPMG train (2), a DC signal is obtained as long as the influence of relaxation is negligible. This indicates that the quantum mechanical propagator describing the echo train commutes with the initial state of the spins. At $2 \mathrm{kHz}$ offset, the decay is also solely governed by relaxation, highlighting the compensational properties of the CPMG sequence. An oscillation of the echo amplitude, as described below, is not visible for an echo delay of $1.5 \mathrm{~ms}$, offset of $2 \mathrm{kHz}$, and RF field intensity of $33.3 \mathrm{kHz}$, because the amplitude variation is approximately $0.1 \%$ (13) — one order of magnitude below the experimental error associated with a single spin-echo amplitude. To reduce the deleterious effects of pulse imperfections in $\mathrm{CP}$ sequences the rectangular hard power pulses were re- placed by HS pulses (sweep width $=10 \mathrm{kHz}$; pulse duration, $t_{\mathrm{p}}=1 \mathrm{~ms}$; adiabacity factor on-resonance, $Q_{0} \approx 9.6(35)$ ). Depending on the relative phase of the AFP-MSE train and on the offset of the AFP pulses, the echo amplitude was reduced by up to $40 \%$ with respect to the standard CPMG sequence (Fig. 1B). With regard to the very fast decay in conventional CP sequences this demonstrates that the HS pulses are far less prone to pulse imperfections and RF field inhomogeneity than hard power rectangular pulses. For the AFP-MSE train applied on-resonance and with relative phase $x$-the AFP-CP sequence-the signal decay was equal to about that for the standard CPMG sequence. On the other hand, when applied with relative phase $y$ (AFP-CPMG) the echo amplitude de- 
cayed to about $36 \%$ within 64 DSPEs. Compared to MSE trains employing hard power pulses, a significant improvement is obtained in the way magnetization components parallel and perpendicular to the phase of the refocusing pulses at the beginning of a MSE train are treated. This improved property, together with the significantly reduced sensitivity to pulse imperfections and RF field inhomogeneity, might especially prove useful for imaging experiments. In MRI experiments stimulated echoes which are magnetization components rotated along the static magnetic field by imperfect refocusing pulses and then rotated back into the transverse plane by subsequent pulses lead to severe image artefacts $(6-12)$. For removal of these artefacts the application of gradients has been proposed $(6-8)$. The gradients, however, destroy the phase relationship between the magnetization and the refocusing pulses. As this relationship is pivotal for the compensational mechanism of pulse imperfections in a CPMG train, this MSE train is no longer a pure CPMG sequence with its favorable properties; magnetization components perpendicular to the phase of the refocusing pulses decay rapidly with an increasing number of spin-echoes (Fig. 2A). To account for this part of the decay, which is not governed by $T_{2}$ relaxation, one has to deconvolute the decay with a weightening function. This weightening function also has to take into account the oscillatory behavior of the decay of the magnetization component of interest (7). In AFPMSE sequences, on the other hand, far more perfect $180^{\circ}$ rotations are obtained. Therefore the magnetization component of interest, i.e., the one which should be perfectly refocused during every spin-echo, decreases mostly due to $T_{2}$ relaxation and stimulated echoes are significantly reduced, thereby avoiding the need for gradients. If a large number of spin-echoes are required the slightly enhanced decay can be taken into account by a weightening function which has to be determined prior to the experiment.

To explore the source of the off-resonance dependence of the decay rate apparent in Fig. 2B the off-resonance profile for an AFP-CPMG train consisting of four double spin-echoes was simulated (Fig. 3A, upper panel). Unexpectedly, not a constant $I_{x}$ coherence is retained but the size of $I_{x}$ oscillates for increasing offsets with increasing amplitude. For comparison, the off-resonance profile of $I_{x}$ for a single double spin-echo is also shown. Oscillations with the same frequency are already visible, although with smaller amplitude. Therefore only for certain, discrete resonance offsets is a pure DC signal obtained (neglecting relaxation), whereas for all other offsets the magnetization decreases with increasing number of spin-echoes. The oscillations of $I_{x}$ with respect to offset are mainly due to an oscillating amount of $I_{z}$ magnetization which is caused both by an imperfect adiabacity of the HS pulse and by the fact that the effective field $\mathbf{B}_{\text {eff }}$ at the beginning of the frequency sweep is not perfectly aligned along the $z$-axis $\left(\Varangle \Theta\left(\mathbf{B}_{\text {eff }}, \mathbf{z}\right) \neq 0^{\circ}\right)$. When changing the cutoff level of the HS pulse to $0.07 \%$ ( $\beta=$ 8 ) and sweeping over $90 \mathrm{kHz}$ with a maximal RF field intensity of $30 \mathrm{kHz}$-resulting in a deviation of $\Theta$ from $0^{\circ}$ by less than $0.05 \%$ and an adiabacity factor on-resonance $Q_{0}>5$-the magnitude of the oscillation of $I_{z}$ could be substantially reduced in the simulations. However, employing this HS pulse in the measurement of spin-echo amplitude profiles did not result in a decay solely governed by $T_{2}$ relaxation pointing to an experimental source of imperfection of the AFP pulses (data not shown). Investigations concerning these imperfections are currently under progress.

Although an accelerated decay apparent in Fig. 2B might be acceptable for some imaging experiments, a MSE sequence is desirable in which the decay of magnetization components of arbitrary phase is exclusively governed by $T_{2}$ relaxation, is insensitive to RF field inhomogeneity, and works well for very large spectral widths. Incorporating the AFP-DSPEs into a MLEV-4 cycle results in such a sequence. From the theory of broadband decoupling we know that applying a MLEV-4 scheme to an inversion element $R$ induces perfect decoupling in cases when $R^{2}$ is the identity operator irrespective of resonance offset (for the approximation regarding only the zerothorder average Hamiltonian) $(31,36$ ). As two sequentially applied, identical AFP pulses act as an identity operator within a frequency range of up to $80 \%$ of the width of the frequency sweep, we applied a MLEV-4 cycling scheme to the AFPDSPEs. This was further encouraged by the simulations of Fig. $3 \mathrm{~A}$ (upper panel), which proved that an $I_{z}$ component generated during the MSE train is responsible for the enhanced decay rates thereby suggesting the combination of $C=R R R R$ with the phase-inverted sequence $C^{\text {inv }}(36)$. The compensational mechanism of the AFP-MLEV-4 sequence is demonstrated by the simulated excitation profile of Fig. 3A (lower panel). The amount of $I_{z}$ magnetization generated during the AFP-MSE train is reduced by more than three orders of magnitude, whereas $I_{y}$ is still reduced by about one order of magnitude. No variation of $I_{x}$ with respect to resonance offset is apparent. To further highlight the compensational mechanism the time evolution of a single quantum in-phase coherence during an AFPMLEV-4 sequence was simulated for a two-spin- $\frac{1}{2}$ system. Both chemical shift evolution and divergence of spin vectors due to scalar coupling were included. Figure $3 \mathrm{~B}$ verifies that both chemical shift evolution and scalar coupling effects are refocused by an AFP-MLEV-4 sequence. Although a varying amount of $I_{z}$ magnetization as well as $I_{z} S_{z}$ longitudinal twospin order is created for a not fully completed MLEV-4 cycle, a very high degree of compensation is obtained at the end of the sequence. These theoretical results were verified experimentally by recording spin-echo amplitude profiles for AFPMLEV-4 sequences on-resonance and at $2 \mathrm{kHz}$ off-resonance both with overall phase $y$ and $x$ of the AFP-MLEV-4 train. Independent of any fixed phase relationship and for large resonance offsets the magnetization decays solely due to irreversible spin-spin relaxation processes (Fig. 2C). Inhomogeneities of the RF field intensity or improper tuning of pulse parameters do not lead to an accelerated decay of signal intensity. The long-term behavior of the AFP-MLEV-4 sequence was compared to various other compensated MSE sequences on-resonance. CPMG, MLEV-16 (37) with overall phase $x$, 


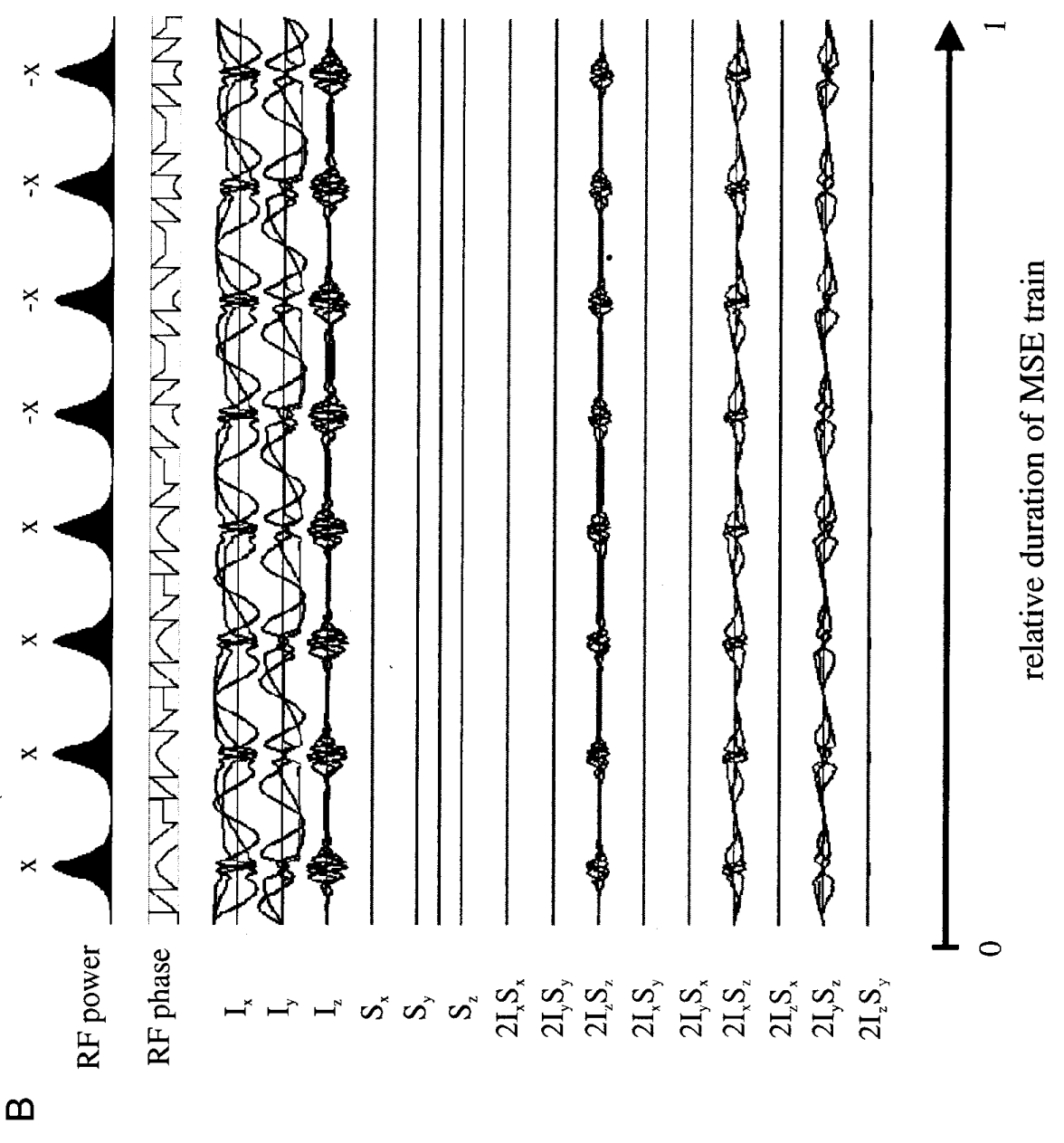

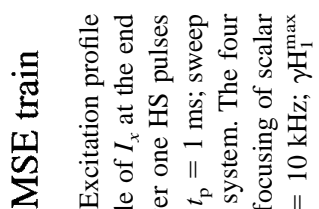

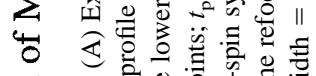



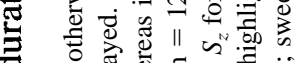

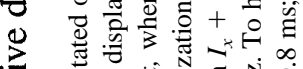

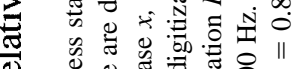

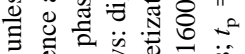



bi

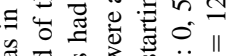

के

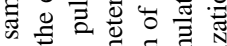

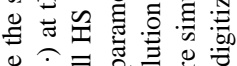

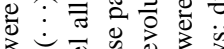

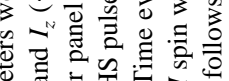

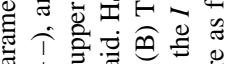

苋 1 势

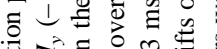



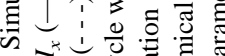
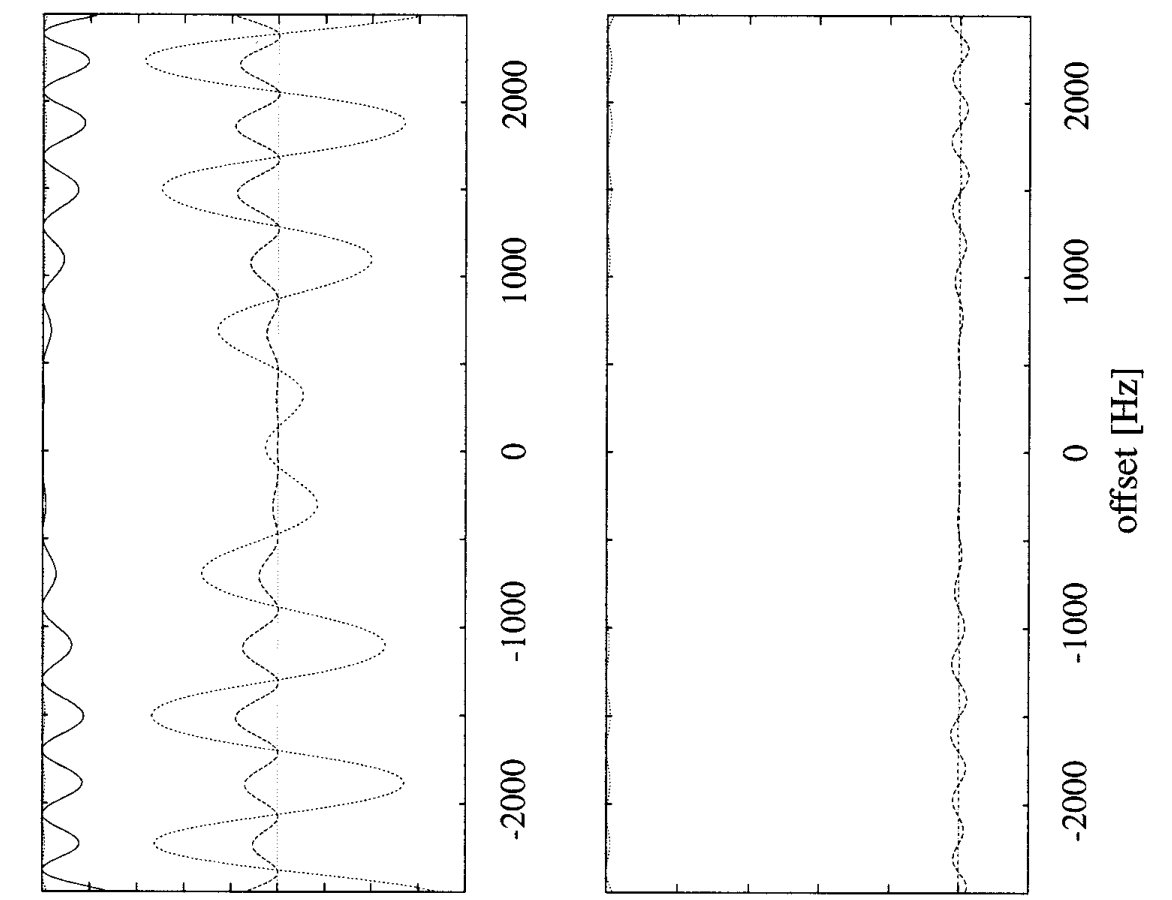

ชิ



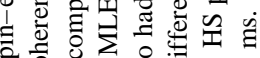

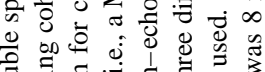

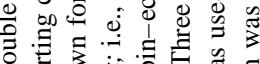

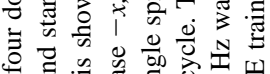

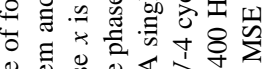

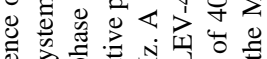

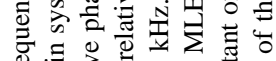


MLEV-16 with overall phase $y$ (data not shown), and XY-16 with overall phase $x(16)$, all with carefully calibrated $180^{\circ}$ pulses, lead to identical decay rates within the experimental errors (Fig. 2D). For the AFP-MLEV-4 sequence a slightly faster decay rate is apparent with an echo amplitude reduced by about 7\% after 352 DSPEs relative to the other MSE sequences. This is due to the residual $y$-component of the magnetization generated during the MSE sequence by minimal imperfections in the AFP pulses that are not completely compensated for by the MLEV-4 cycle (Fig. 3A, lower panel). To compensate these residual deviations from the identity operator, the MLEV-4 cycle was replaced by a MPFn cycling scheme (38) in which each composite pulse was substituted by an AFP-DSPE. With this scheme all residual imperfections could be compensated for (Fig. 2D).

There are several applications for which the preservation of all three components of magnetization is important $(14,16$, 18); excellent inversion of the longitudinal component has been shown by several groups before. These applications include imaging experiments in which utilization of phase-encoding gradients destroys the phase relationship between the magnetization and the refocusing pulses, thereby eliminating the compensational mechanism of the CPMG sequence (14), the measurement of homonuclear dipole couplings in the presence of chemical shifts, and the measurement of heteronuclear dipole couplings in REDOR experiments $(16,18)$. To achieve the preservation of all three magnetization components, different compensated $\mathrm{CP}$ sequences have been proposed (14-16) which all rely on phase alternating schemes applied to hard power refocusing pulses. However, all these MSE sequences require practically at least 16 pulses for proper compensation of pulse imperfections, cause artefacts due to stimulated echoes, and are only applicable for limited resonance offsets. Moreover they are still fairly sensitive to proper tuning of pulse parameters and to the homogeneity of the RF field intensity (11, 14-16). All these drawbacks are eliminated when using AFP-MSE sequences.

\section{Removal of Systematic Errors Associated with Off- Resonance Effects}

A source for deviations from an exponential decay governed solely by the spin-spin relaxation time $T_{2}$, even in the case of perfect $180^{\circ}$ pulses, are off-resonance effects. Due to off-resonance effects the refocusing transverse component of the magnetization is reduced and a longitudinal component is created. Thereby it is especially detrimental if the magnitude of these components is oscillating with resonance offset for successive RF pulses $(12,13)$. This oscillating behavior can be observed in Figs. 4A and 4B for a $\mathrm{CP}$ and a CPMG sequence, respectively. The acquired free induction decays were Fourier transformed both in the acquisition dimension and with respect to the number of repetitions of the double spin-echo elements. Side bands are clearly visible in addition to the exponentially damped center signal with zero frequency in $F_{1}$ (which was suppressed by the application of a filter). Their offsets and amplitudes correspond to the frequencies and magnitudes of these oscillations. The compensational mechanism of CPMG trains reduces the magnitude of side band intensities by about $75 \%$ with respect to CP sequences. Nevertheless significant oscillating components remain as highlighted by various cross sections along $F_{1}$ (Fig. 5B). To evaluate the possibility of suppressing the oscillating behavior by the application of gradients a $(1,0,1, \ldots)$ gradient scheme was incorporated into the CPMG train; i.e., every odd refocusing pulse was surrounded by two equal gradients (7). However, with our experimental conditions, and opposite to observations previously reported (17), we were not able to remove the oscillating behavior of the decay (Fig. 4C). The maximum intensity for the side bands could not be reduced (Fig. 5C right-hand side); only the dependence of the frequency of the oscillating component on resonance offset was altered. This is caused by the fact that because of applied gradients, stimulated echoes are effectively suppressed (6, 7). These stimulated echoes otherwise mask the true dependence of the oscillating component on resonance offset. The increasing frequency of the oscillations for increasing resonance offset is in accordance with the theoretical and experimental results of Majudmar et al. (12). In the same way as gradients failed in the removal of systematic errors associated with off-resonance effects, composite pulses did not result in their suppression $(12,13)$. The offset dependence was only modified; in addition, composite pulses are far more sensitive to RF field inhomogeneity compared to AFP pulses, especially when they are optimized for compensation of frequency offset (23).

In Fig. 4D the pseudo-2D spectrum is presented which was obtained when the rectangular hard power pulses of a $\mathrm{CP}$ train were replaced by HS pulses. Oscillating contributions are almost completely removed. Only very weak residual side bands are visible. The extent of elimination of off-resonance effects is highlighted in Fig. 5D. The maximum intensity of side bands is reduced by two orders of magnitude (Fig. 5D, right-hand side). At $200 \mathrm{~Hz}$ resonance offset the side band intensity amounts to only $0.04 \%$. Moreover the size of the side band intensity at this offset is more than twice as large as that seen at other resonance offsets, indicating that on average one only has to cope with off-resonance contributions of the size of $0.01 \%$. These are almost of a magnitude of residual baseline distortions caused by the applied filter for suppression of the nonoscillating component (Fig. 5D, left-hand side). Moreover, they do not increase with increasing resonance offset but even decrease for higher offsets. The same quality of removal of off-resonance effects was retained when incorporating MLEV-4 cycling into the AFP-MSE train (data not shown). Putting this together with the results of Fig. 2 it demonstrates that oscillating components are not simply destroyed during an AFP-MSE sequence; rather, the creation of an oscillating component is avoided in the first place by the use of HS pulses as refocusing pulses. Figures 4D and 5D demonstrate that the application of AFP pulses as refocusing pulses suppresses the creation of offset-frequency-dependent effects, thereby elimi- 
A

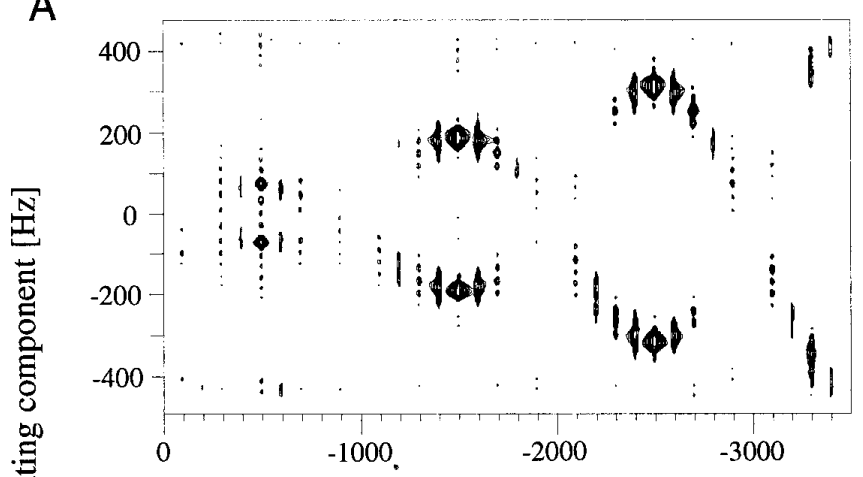

B

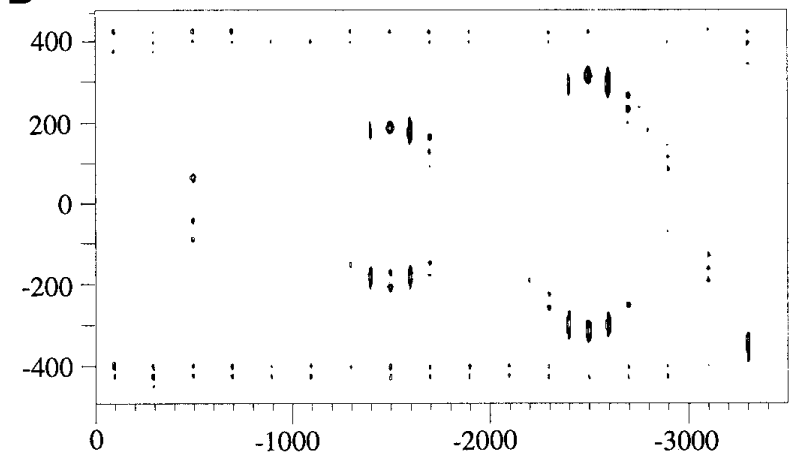

D

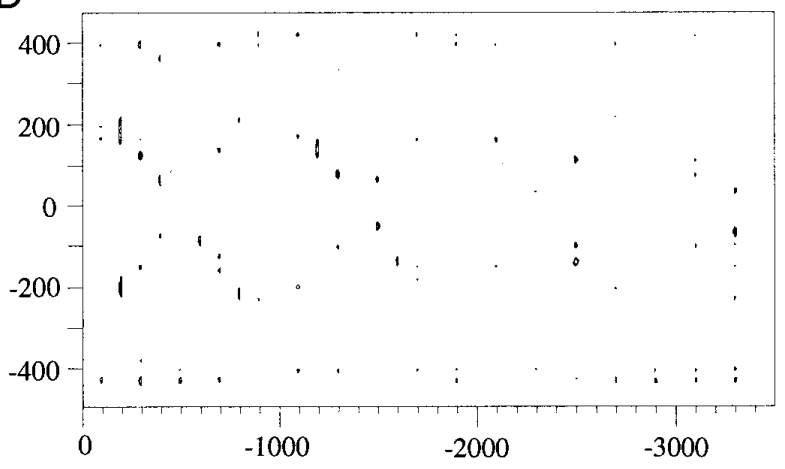

offset $[\mathrm{Hz}]$

FIG. 4. 2D representation of off-resonance effects in MSE trains with a double spin-echo as basic building block. The spectral width shown along $F_{1}$ is $952.4 \mathrm{~Hz}$ given by $\left[\left(2 \Delta+T_{180^{\circ}}\right)\right]^{-1}$. All profiles are displayed at the same contour level. (A) For refocusing, rectangular pulses of length $100 \mu \mathrm{s}$ were used. The de/refocusing delay $\Delta$ was $475 \mu$ s. $180^{\circ}$ refocusing pulses were applied with the same phase as the excitation pulse (CP scheme). (B) Same as (A) but CPMG sequence. (C) CPMG train combined with a $(1,0,1, \ldots)$ gradient scheme (7). (D) $\mathrm{CP}$ train with HS pulses $\left(t_{\mathrm{p}}=360 \mu \mathrm{s}\right.$, sweep width $=20 \mathrm{kHz}, \gamma \mathrm{H}_{1}^{\text {max }}=24.5$ $\mathrm{kHz}$, digitization $=512$ points) replacing the rectangular pulses.

nating the need for any correction scheme or for careful adjustment of echo delays.

\section{Measurement of $T_{2}$ Relaxation Times Employing AFP- MLEV-4 Trains}

The pulse sequences used for measurement of $T_{2}$ relaxation times of segment 4 of the rod domain of the gelation factor from $D$. discoideum (32) are presented in Fig. 6. A ${ }^{1} \mathrm{H}-{ }^{15} \mathrm{~N}$ shift correlation spectrum recorded with the sequence of Fig. $6 \mathrm{~B}$ is displayed in Fig. 7. It demonstrates that a high quality spectrum with no phase roll along the ${ }^{15} \mathrm{~N}$ dimension can be acquired by an AFP-MLEV-4 sequence. The absence of any phase distortion in the indirect frequency dimension is further highlighted by the cross section at $\delta\left({ }^{1} \mathrm{H}\right)=9.23 \mathrm{ppm}$. This cross section shows two purely absorptive signals, the signals of Gly 12 and Lys 25 of segment 4, having a chemical shift difference of $\Delta \delta\left({ }^{15} \mathrm{~N}\right)=15.31 \mathrm{ppm}$. The $T_{2}$ relaxation times measured with the standard pulse sequence and with the AFP version were evaluated for a few resonances and compared to each other. Resonances were chosen which were on-resonance or almost on-resonance and therefore have peak heights free from systematic errors when measured with the standard pulse sequence. The decay of signal intensity with increasing relaxation delay $\mathrm{T}$ for Phe 24 determined both with the standard sequence and with the one employing an AFP-MLEV-4 train is shown in Fig. 8A. The decreasing peak heights of Phe 24 measured with the two different pulse sequences are equal within the experimental errors. Analogous results were obtained for the resonances of Glu 4, Ser 6, Glu 9, Glu 73, and Thr 82. The $T_{2}$ relaxation times of these resonances, their corresponding errors, and the residual $\chi^{2}$ values as measures for the quality of the monoexponential fit are listed in Table 1 . Within the errors determined by Monte-Carlo simulations, the ${ }^{15} \mathrm{~N} T_{2}$ relaxation times determined with the pulse sequence employing an AFP-MLEV-4 train are equal to those obtained using a standard CPMG spin-echo train. In both cases residual $\chi^{2}$ values of the fits are below 7.82 for each resonance; 7.82 is the critical value for three degrees of freedom of the exact $\chi^{2}$ statistic at a 5\% level of significance (39). Therefore, the $T_{2}$ decay curve of each single resonance obtained using an AFPMLEV-4 train can be adequately described by a monoexponential model function.

To demonstrate the improved properties with respect to off-resonance effects for the AFP-MLEV-4 sequence a series 



frequency of oscillating component $[\mathrm{Hz}]$

FIG. 5. Cross sections along $F_{1}$ from the off-resonance profiles in Fig. 4 at a resonance offset of $2.6 \mathrm{kHz}$ (left) and at resonance offsets for which maximal side band intensity was observed (right). On the right-hand side the maximal size of the side band intensity (within a resonance offset of $3.4 \mathrm{kHz}$ ) relative to the on-resonance intensity (average value calculated from signals at resonance offsets free from systematic errors) is indicated for each MSE sequence. The asymmetry visible especially in (A) and (B) of the left-hand side is introduced by baseline distortions from a neighboring line.

of six 2D spectra employing the sequence of Fig. 6B and stepping the offset of the AFP pulses from 0 to $2000 \mathrm{~Hz}$ were recorded. Relative deviations in peak heights from the average value with respect to offset were determined (Fig. 8B). Within the experimental error of about $2 \%$, given by the root-meansquare baseline noise, no variation of peak intensities for increasing resonance offset is apparent. The limiting factor for the usable spectral width in the ${ }^{15} \mathrm{~N}$ dimension is determined by the type of HS pulses employed. A few factors have to be considered. First, what is the maximal RF field intensity available for the ${ }^{15} \mathrm{~N}$ nucleus? Second, how long may the duration of de/refocusing delays be chosen to avoid significant evolution of antiphase magnetization with its accelerated $T_{2}$ relaxation? Third, which maximal duration of the HS pulse relative to the de/refocusing delays allows measurement of $T_{2}$ times without influence of $T_{1}$ active during some part of the AFP pulses (Fig. 1A)? Fourth, which width of the frequency sweep is possible for the parameters determined by the above factors and still results in a HS pulse of sufficient adiabacity? Factor one is determined by the spectrometer-in our case $\gamma H_{1}^{\max }=$ $6.95 \mathrm{kHz}$. From previously reported investigations (40) it is known that a duration of $0.5 \mathrm{~ms}$ of the defocusing delay results in errors less than $1 \%$ in measured $T_{2}$ times. For such a duration the length of the HS pulse can be set to at least $800 \mu \mathrm{s}$ without introducing significant errors in measured $T_{2}$ times. This is apparent from the values of Glu 9 listed in Table 1. Glu 9 has a $T_{1}$ of $1.1 \mathrm{~s}$, about 1.5 times as long as the average $T_{1}$ over the whole sequence of the gelation factor, but still gives the same $T_{2}$ times-compared to conventional sequenceswhen measured with the AFP-MLEV-4 sequence of Fig. 6B. As in our investigations an on-resonance adiabacity factor $Q_{0}$ of 0.6 for the HS pulses was sufficient when they were incorporated into an AFP-MLEV-4 train, because the frequency of the HS pulse can be swept over $10 \mathrm{kHz}$, resulting in a sufficiently large, usable spectral width in the ${ }^{15} \mathrm{~N}$ dimension. From these considerations it becomes clear that the higher the maximal RF field intensities available the larger the spectral width for which the AFP-MLEV-4 train results in error free measurements of $T_{2}$ relaxation times. Similarly, when ${ }^{13} \mathrm{C} T_{2}$ relaxation times were to be measured the larger spectral widths necessary can also be covered, as higher maximal RF field intensities are available for ${ }^{13} \mathrm{C}$ nuclei.

We did not encounter any problems with sample heating for the parameters of the AFP-MLEV-4 train used in our investigations. The amount of power deposition by the AFP pulses can be further reduced when ordinary HS pulses are replaced by AFP pulses that are optimized for minimal power deposition such as, for example, HS-8 (41). In the pulse sequence of Fig. $6 \mathrm{~B}$ the rectangular $180^{\circ}$ pulses during the INEPT, the reverse INEPT and the rectangular ${ }^{1} \mathrm{H} 180^{\circ}$ pulse during the evolution time $t_{1}$ can also be replaced by AFP pulses. However, this did not result in any improvement for the ${ }^{15} \mathrm{~N} T_{2}$ relaxation time measurements performed in this study. On the other hand, for ${ }^{13} \mathrm{C} T_{2}$ relaxation time measurements replacement of the rectangular $180^{\circ}$ pulses on ${ }^{13} \mathrm{C}$ by AFP pulses might be necessary in order to avoid signal reduction for resonances at large frequency offsets. Also, complications associated with Hartmann-Hahn transfers (42) expected in $T_{1 \rho}$ experiments on ${ }^{13} \mathrm{C}^{\alpha}$ resonances of residues that have the ${ }^{13} \mathrm{C}^{\beta}$ resonances nearby should be removed. In general, the measurement of $T_{2}$ relaxation times for basically unlimited frequency offsets without the necessity for any correction scheme should be the main 


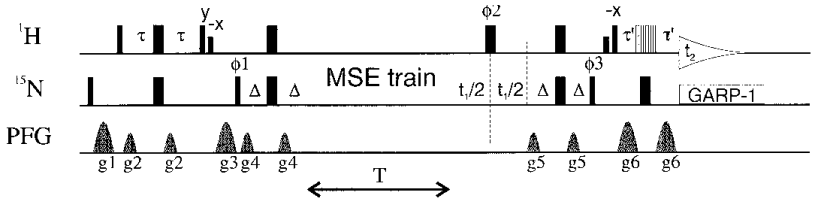

A



B

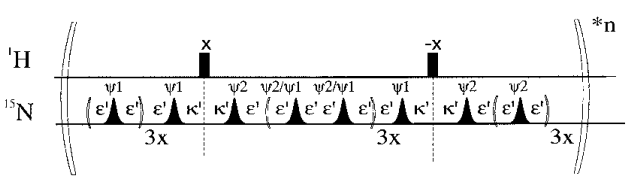

the measurement of $T_{2}$ relaxation times without systematic errors due to pulse imperfections, RF field inhomogeneity, or off-resonance effects. Therefore, degradation of image quality in MRI is also avoided. Since all three magnetization components are treated equally during an AFP-MLEV-4 train, application of AFP pulses should be beneficial to NMR experiments that rely on the premise that two sequentially applied refocusing elements act as a true identity operator irrespective of the direction of the magnetization. At the same time careful pulse calibration is not required. The usage of an AFP-MLEV-4 train in standard pulse sequences for the determination of ${ }^{15} \mathrm{~N}$ $T_{2}$ relaxation times resulted in error-free results, demonstrating that it is advantageous to use AFP-MSE trains in $T_{2}$ relaxation time measurements.

\section{EXPERIMENTAL}

FIG. 6. Pulse sequences for the measurement of ${ }^{15} \mathrm{~N} T_{2}$ relaxation times. In all sequences narrow and wide rectangles indicate hard power $90^{\circ}$ and $180^{\circ}$ pulses, respectively. The ${ }^{1} \mathrm{H}$ hard power pulses had a RF field intensity of $\gamma \mathrm{H}_{1}$ $=23.6 \mathrm{kHz},{ }^{15} \mathrm{~N}$ hard power pulses of $\gamma \mathrm{H}_{1}=6.95 \mathrm{kHz}$. Smaller rectangles represent rectangular water flip-back pulses with a RF field intensity $\gamma \mathrm{H}_{1}=$ $220 \mathrm{~Hz}$. Pulsed field gradients are shown in grey. Unless indicated otherwise, all pulses are applied along the $x$-axis. For suppression of the solvent signal a WATERGATE sequence was employed (53). In both experiments the delays were set as follows: $\tau=2.4 \mathrm{~ms}\left(<1 / 4 J_{\mathrm{NH}}\right), \Delta=2.75 \mathrm{~ms}\left(=1 / 4 J_{\mathrm{NH}}\right), \tau^{\prime}=$ $1.05 \mathrm{~ms} .{ }^{15} \mathrm{~N}$ decoupling during acquisition was achieved with a GARP-1 sequence (54) using a $0.96-\mathrm{kHz}$ RF field. Gradients were applied along $z$, were sine shaped, and had the following relative strengths and durations: $g_{1}=$ $-43 \%, 1 \mathrm{~ms} ; g_{2}=15 \%, 1 \mathrm{~ms} ; g_{3}=-90 \%, 3 \mathrm{~ms} ; g_{4}=25 \%, 1 \mathrm{~ms} ; g_{5}=33 \%$, $1 \mathrm{~ms} ; g_{6}=80 \%, 1 \mathrm{~ms}$. The maximum absolute gradient strength was approximately $50 \mathrm{G} / \mathrm{cm}$. For frequency discrimination in the indirect dimension the STATES-TPPI method was applied (55). Phase cycling employed in both experiments was $\phi_{1}=x,-x ; \phi_{2}=y, y,-y,-y ; \phi_{3}=x, x,-x,-x$; receiver $=x,-x,-x, x$. The CPMG sequence (2) was expanded according to Kay et al. (40) and Palmer et al., (56) by incorporating ${ }^{1} \mathrm{H} 180^{\circ}$ pulses every $4 \mathrm{~ms}$. To avoid significant influence of relaxation of antiphase magnetization $(48,49)$ in $(\mathrm{A}) \epsilon=0.5 \mathrm{~ms}-0.5 \mathrm{pwn}$ and $\kappa=\epsilon-0.5 \mathrm{pw}$ were chosen where pwn and pw are the durations of the $180^{\circ}{ }^{15} \mathrm{~N}$ and ${ }^{1} \mathrm{H}$ pulse, respectively. In (B) the AFP-MLEV-4 sequence is shown which removes systematic errors due to off-resonance effects and pulse imperfections. The hard power $180^{\circ}{ }^{15} \mathrm{~N}$ pulses of the standard CPMG train are replaced by HS pulses $\left(t_{\mathrm{p}}=0.8 \mathrm{~ms}\right.$, sweep width $=10 \mathrm{kHz}, \gamma \mathrm{H}_{1}^{\max }=6.95 \mathrm{kHz}$, digitization $=1024$ points $)$ and a MLEV-4 cycle is applied to the DSPEs. The MLEV-4 cycling is indicated with the phases $\psi_{1}=x$ and $\psi_{2}=-x$. The de/refocusing times have been adjusted to $\epsilon^{\prime}=0.5 \mathrm{~ms}-0.5 t_{\mathrm{p}}$ and $\kappa^{\prime}=\epsilon^{\prime}-0.5 \mathrm{pw}$ to have the same duration of the spin-echoes as in (A).

advantage compared to the spin-lock technique proposed by Peng et al. $(43,44)$. In addition, no reduction of signal amplitude due to defocusing of magnetization components not aligned parallel to the applied RF field arises compared to the spin-lock technique.

\section{CONCLUSIONS}

Spectral phase rolls caused by single AFP pulses used as refocusing elements are removed for even numbers of spinechoes; the quality of this removal with modern hardware is such that AFP pulses incorporated into MLEV-4 cycles allow
All NMR experiments were performed on a Bruker DRX 600 spectrometer equipped with a triple-resonance $\left({ }^{1} \mathrm{H} /{ }^{15} \mathrm{~N} /\right.$ ${ }^{13} \mathrm{C}$ ) three-axes gradient probehead (TXI X/Y/Z-grad) operated at $300 \mathrm{~K}$. In order to avoid modulation effects the samples were not spun. To exclude radiation damping and to have a single signal spectrum with high signal-to-noise ratio a $600-\mu l$ sample of $1 \% \mathrm{H}_{2} \mathrm{O}$ in $\mathrm{D}_{2} \mathrm{O}$ doped with $\mathrm{CuSO}_{4}(2 \mathrm{mM})$ was utilized for measurement of refocusing, echo amplitude, and off-resonance profiles. The AFP-MSE sequences were tested on a sample containing approximately $2.1 \mathrm{mM}$ of uniformly ${ }^{15} \mathrm{~N}$-labeled segment 4 of the rod domain of the gelation factor from $D$. discoideum dissolved in $90 \% \mathrm{H}_{2} \mathrm{O} / 10 \% \mathrm{D}_{2} \mathrm{O}$ at $\mathrm{pH}=7$ (32). The data were processed and analysed using the programs XWINNMR (45) and CCNMR (46). The freeware program GNUPLOT was used to display data curves. The amplitude and phase profiles of the HS pulses for both the simulation and NMR experiments were generated with $\mathrm{C}$ programs and then transformed to binary files using the xShape tool of Bruker Analytische Messtechnik GmbH, Karlsruhe, Germany. Simulations were performed with the program PENCIL (47).

Refocusing profiles of Fig. 1B were acquired by single-scan spin-echo (upper panel) or double spin-echo (lower panel) sequences in which the echoes were surrounded by a $z$-gradient pair. The two gradients had equal strength (within the hardware specifications) of about $40 \mathrm{G} / \mathrm{cm}$ and had either same (spinecho) or opposite (double spin-echo) signs. In both cases the recovery delay was $5 \mathrm{~s}$ and the rectangular excitation pulse had a RF field intensity of $\gamma \mathrm{H}_{1}=33.3 \mathrm{kHz}$. Unless stated otherwise, all HS pulses had a $1 \%$ cutoff level. Refocusing profiles for various echo delay durations ranging from 0.32 to $10.32 \mathrm{~ms}$ were recorded. The HS pulses had a duration of $1 \mathrm{~ms}$, a frequency sweep width of $10 \mathrm{kHz}$, and a maximum RF field intensity of $\gamma \mathrm{H}_{1}^{\max }=33.3 \mathrm{kHz}$ and were digitized with $1 \mathrm{~K}$ points. To avoid phase problems induced by transmitter jumps between different offsets, resonance offsets were created by adding a phase ramp to the original phase-modulation function. The on-resonance spectrum was phased to absorption mode and this phase correction was applied to all other traces. 


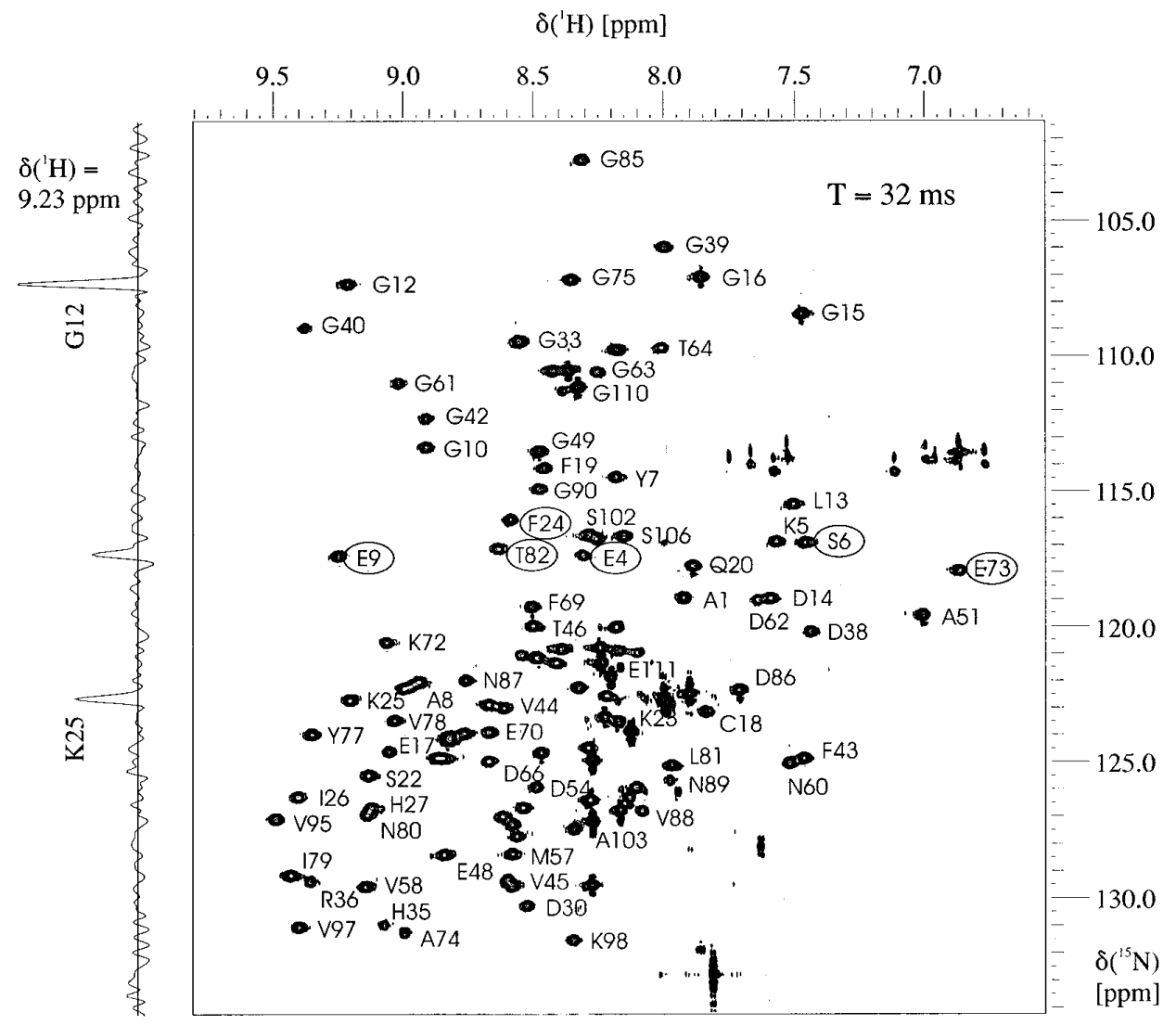

FIG. 7. ${ }^{1} \mathrm{H}-{ }^{15} \mathrm{~N}$ shift correlation spectrum of segment 4 of the rod domain of the gelation factor (31) recorded with the AFP-MLEV-4 pulse sequence of Fig. 6B, employing a relaxation delay $T=32 \mathrm{~ms}$. Signals from backbone amide resonances are labeled with one-letter abbreviations of the amino acid type and the corresponding sequence number. Resonances for which $T_{2}$ decay curves were evaluated are marked with a circle. For Gly 12 the $F_{1}$ cross section at $\delta\left({ }^{1} \mathrm{H}\right)$ $=9.23 \mathrm{ppm}$ is shown.

Echo amplitudes for increasing number of double spinechoes were acquired with the parameters given in the legend of Fig. 2. The $4 \mathrm{~K}$ data points for a sweep width of $400 \mathrm{~Hz}$ were recorded for each single number of double spin-echoes and multiplied by an exponential window function of $0.3 \mathrm{~Hz}$ prior to Fourier transformation. The resulting signal was baselinecorrected and integrated in order to obtain the magnitude of the echo amplitude. For determination of the experimental error of a single echo amplitude the standard CPMG train was recorded twice and the difference in amplitude for each single number of DSPEs was calculated. The standard deviation of these differences times $2 * 1 / \sqrt{2}$ was finally taken as an estimate for the experimental error of a single echo amplitude. The same procedure was performed for the AFP-MLEV-4 train. For the AFP-MLEV-4 train the standard deviation of the differences was $10 \%$ higher than for the standard CPMG sequence. Therefore for all MSE sequences utilizing AFP pulses this higher value was used as an estimate for the error.

The 2D spectra in Figs. 4A and 4B demonstrating the existence of off-resonance effects in CP and CPMG sequences were recorded and processed as described by Ross et al. (13). The quality of removal of off-resonance effects with gradients (Fig. 4C) was investigated by incorporating the $(1,0,1, \ldots)$ gradient scheme in a CPMG train, i.e., placing every odd refocusing pulse between two equal gradients (12). The square-shaped gradients had a duration of $230 \mu \mathrm{s}$ and a strength of $1 \mathrm{G} / \mathrm{cm}$. The spectrum in Fig. 4D was recorded in an analogous way to those in Figs. 4A and 4B; the de/refocusing delay was adjusted to $345 \mu$ s to account for the longer duration of the HS pulse.

The $T_{2}$ relaxation times were determined with pulse sequences based on a sequence reported by Farrow et al. (48) which causes only minimal saturation of water. As no sensitivity enhancement was used, an additional selective pulse on water had to be used in order to return water to the $z$-axis prior to data acquisition. $T_{2}$ decay curves were sampled at relaxation delays of both 16, 32, 64, 96, and $160 \mathrm{~ms}$ and 19.2, 38.4, 76.8, 115.2 , and $192 \mathrm{~ms}$. These sampling points allow a two-parameter fit to the decay curve (49). For the sequence in which the phase of double refocusing elements was modulated according to MLEV-4 cycling HS pulses digitized with $1 \mathrm{~K}$ points and of $0.8 \mathrm{~ms}$ duration, $10 \mathrm{kHz}$ sweep width and $6.95 \mathrm{kHz}$ maximal RF field intensity were used. The $2 \mathrm{D}$ spectra were acquired in an interleaved manner, i.e., data were acquired for all five time points before $t_{1}$ incrementation, to minimize effects both due to spectrometer drift and heating. Total measurement time for a $T_{2}$ experiment with five sampling points was about $5 \mathrm{~h}$. Single 


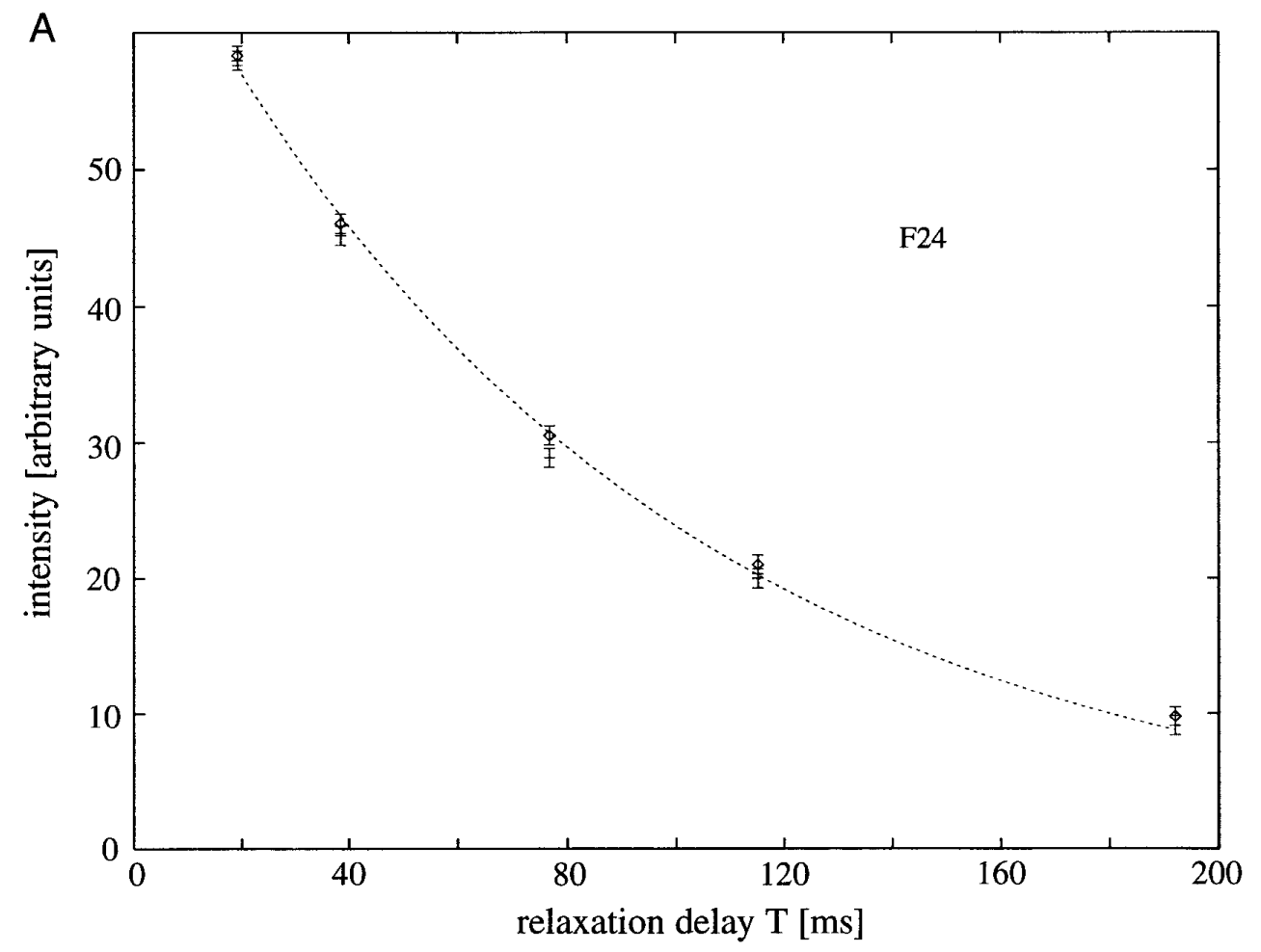

$\mathrm{B}$

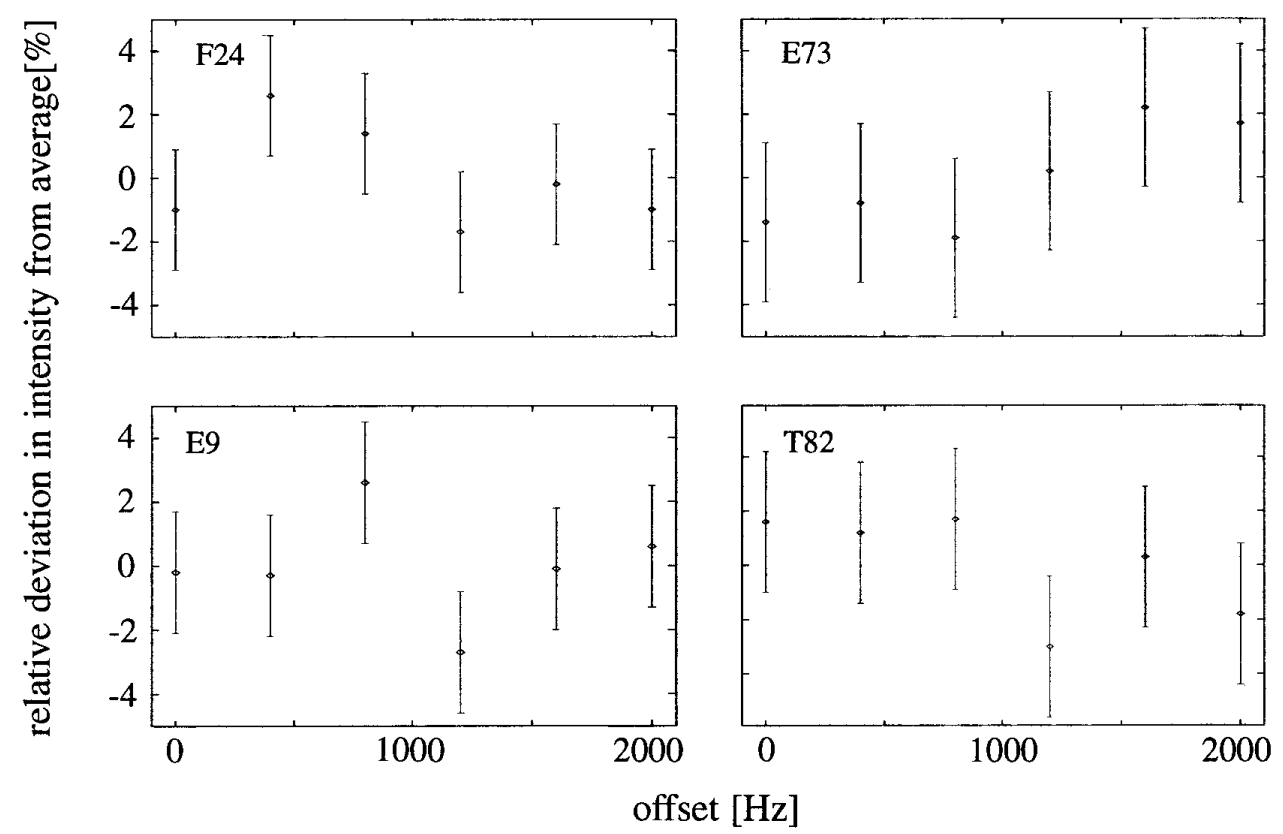

FIG. 8. (A) Comparison of the decay of signal intensity with increasing relaxation delay $T$ for Phe 24 of segment 4 of the gelation factor (see Fig. 7) measured with the standard pulse sequence of Fig. 6A $(\diamond)$ and with the pulse sequence utilizing an AFP-MLEV-4 train (Fig. 6B) (+). To highlight the exponential behavior of the decays the fit curve of the AFP-MLEV-4 train is displayed. Errors of peak intensities were determined as described in the text. (B) Relative deviation in peak intensity from average value with respect to offset of the HS pulses for Glu 9, Phe 24, Glu 73, and Thr 82. Offsets were created by addition of an appropriate phase ramp to the original phase-modulation function.

2D spectra were recorded as $64 * \times 1 \mathrm{~K}^{*}$ data matrices with 16 scans for each $t_{1}$ value and a spectral width of $32.9 \mathrm{ppm}$ in $F_{1}$ and $11.0 \mathrm{ppm}$ in $F_{2}$. The improved properties with respect to off-resonance effects for the newly proposed AFP-MLEV-4 train when used for ${ }^{15} \mathrm{~N} T_{2}$ measurements were demonstrated by recording a series of six $2 \mathrm{D}$ spectra employing the sequence of Fig. 6B and stepping the offset of the AFP pulses from 0 to $2000 \mathrm{~Hz}$ (Fig. 8B). A relaxation delay of $96 \mathrm{~ms}$ was used (6 
TABLE 1

${ }^{15} \mathrm{~N} T_{2}$ Relaxation Times, Their Corresponding Errors, and the Residual $\chi^{2} \mathrm{~V}$ alues

\begin{tabular}{|c|c|c|c|c|c|c|c|}
\hline \multirow[b]{2}{*}{ Amino acid } & \multirow{2}{*}{$\begin{array}{c}\text { Frequency offset } \\
\delta\left({ }^{15} \mathrm{~N}\right)[\mathrm{Hz}]\end{array}$} & \multicolumn{3}{|c|}{ Standard CPMG train } & \multicolumn{3}{|c|}{ AFP-MLEV-4 train } \\
\hline & & $T_{2}[\mathrm{~ms}]$ & \pm error of $T_{2}[\mathrm{~ms}]$ & Residual $\chi^{2}$ & $T_{2}[\mathrm{~ms}]$ & \pm error of $T_{2}[\mathrm{~ms}]$ & Residual $\chi^{2}$ \\
\hline E4 & 50 & 67.7 & 6.3 & 1.0 & 72.0 & 7.4 & 7.7 \\
\hline S6 & 76 & 109.3 & 3.1 & 1.9 & 103.2 & 2.7 & 2.0 \\
\hline E9 & 39 & 102.1 & 10.5 & 3.1 & 103.5 & 10.7 & 2.9 \\
\hline F24 & 117 & 97.2 & 9.9 & 3.1 & 95.6 & 3.2 & 2.5 \\
\hline E73 & 0 & 71.9 & 7.5 & 0.6 & 73.9 & 7.6 & 5.8 \\
\hline T82 & 55 & 91.4 & 3.0 & 3.0 & 93.9 & 2.4 & 1.1 \\
\hline
\end{tabular}

times the basic MSE block shown in Fig. 6B). AFP pulses were HS shaped, digitized with $1 \mathrm{~K}$ points, of $0.8 \mathrm{~ms}$ duration, 10 $\mathrm{kHz}$ sweep width, and $6.95 \mathrm{kHz}$ maximal RF field intensity. Each $2 \mathrm{D}$ spectrum was recorded as $128^{*} \times 2 \mathrm{~K} *$ data matrix with 16 scans and a recovery delay of $3 \mathrm{~s}$ to obtain high signal-to-noise ratio and avoid the need for linear prediction, thereby keeping the uncertainties in the peak intensities at a minimum (50).

The $T_{2}$ relaxation time experiments were processed and analyzed with the program CCNMR (46). For determination of decay curves and $T_{2}$ relaxation times two-dimensional Lorentzian functions were fitted to the signals in the spectrum with the shortest relaxation delay. In the spectra with increased relaxation delays positions and line widths were not changed, and only the amplitude of the two-dimensional Lorentzian functions was fit to obtain peak intensities. Peak heights were chosen for cross peak quantification, as they are known to be more accurate then peak volumes $(50) .{ }^{1} \mathrm{H}-{ }^{15} \mathrm{~N}$ shift correlation spectra recorded as $64 * \times 1 \mathrm{~K}^{*}$ data matrices were apodized with pure exponential functions, linear predicted up to $128^{*}$ data points in $F_{1}$, zero filled to $256^{*} \times 2 \mathrm{~K}^{*}$ data points, and baseline corrected in both dimensions. Those measured as $128^{*} \times 2 \mathrm{~K}^{*}$ data matrices were processed similarly; however, no linear prediction was applied.

Uncertainties of measured peak heights were determined by a three step process. First, a Monte-Carlo-type simulation was performed in order to account for the frequency distribution of the baseline noise, which has to be taken into account when a fit procedure is used. In this simulation the uncertainty of the intensity of a single peak is determined by shifting the corresponding "modeled" peak, i.e., the one obtained by the fit procedure, to positions in an area where no peaks are found and by refitting this peak for each new position. The refitting of the modeled peak was performed for 100 different positions. The standard deviation of the 100 measured intensity values was taken as a first estimate for the experimental uncertainty of the measured peak heights. The same procedure was employed for each signal in the ${ }^{1} \mathrm{H}-{ }^{15} \mathrm{~N}$ shift correlation spectrum with the shortest relaxation delay in order to account for different peaks heights. Furthermore, duplicate ${ }^{1} \mathrm{H}-$ ${ }^{15} \mathrm{~N}$ shift correlation spectra with $T=16 \mathrm{~ms}$ and for the $128^{*} \times$ $2 \mathrm{~K}^{*}$ data matrices with $T=96 \mathrm{~ms}$ were recorded with the sequence of Fig. 6B. The standard deviation of the differences between the heights of corresponding peaks in the two spectra multiplied by $1 / \sqrt{2}$ (assuming identical distributions of the peak heights of the amide backbone resonances) was compared to the estimated errors determined with the procedure described above and to the root-mean-square baseline noise in the spectra. The root-mean-square baseline noise was determined in spectra employing different relaxation delays. Similar to observations already reported $(49,50)$, a decrease of root-mean-square baseline noise with increasing relaxation delay was found (10-15\% less at a relaxation delay $T=192 \mathrm{~ms}$ compared to $T=19.2 \mathrm{~ms}$ ). Uncertainties in measured peak heights were finally set equal to the root-mean-square baseline noise of the spectra with the shortest relaxation delay, as this was the largest estimate for the experimental error.

From decaying peak heights and their uncertainties $T_{2}$ relaxation times and their corresponding errors were obtained by a $\chi^{2}$ minimization combined with a Monte-Carlo simulation in a way similar to the ones described by Kamath et al. (51) and Palmer et al. (52). Instead of using a two-parameter nonlinear optimization, however, a combination of a one-dimensional grid search for the optimal decay constant and a linear fit of the signal amplitude at $T=0 \mathrm{~ms}$ was used. A goodness-of-fit test with the residual $\chi^{2}$ value as test statistic (39) was utilized to proof the monoexponential behavior of signal decays for both the standard sequence and the pulse sequence employing an AFP-MLEV-4 train.

\section{ACK NOWLEDGMENTS}

The authors thank Christian Renner for stimulating discussions and careful reading of the manuscript. We are grateful to Christian Cieslar for support in handling the program CCNMR and to Paola Fucini for supplying the sample of the gelation factor. M. Z. was supported by a scholarship of the Verbandes der Chemischen Industrie e. V.

\section{REFERENCES}

1. H. Y. Carr and E. M. Purcell, Phys. Rev. 94, 630 (1954).

2. S. Meiboom and D. Gill, Rev. Sci. Instrum. 29, 688 (1958).

3. C. E. Mills, L. E. Crooks, L. Kaufman, and M. Brandt-Zawadski, Radiology 150, 87 (1984).

4. S. J. Riederer, S. A. Suddarth, S. A. Bobman, J . N. Lee, H. Z. Wang, and J. R. Macfall, Radiology 153, 203 (1984).

5. L. E. Kay, D. A. Torchia, and A. Bax, Biochemistry 28, 8972 (1989). 
6. J. H. Duijn, J. H. N. Creyghton, and J. Smidt, Scientific Program from the Third Annual Meeting of the Society of Magnetic Resonance in Medicine, New York, p. 197, 1984.

7. S. Majumdar, S. C. Orphanoudakis, A. Gmitro, M. O'Donnell, and J. C. Gore, Magn. Reson. Med. 3, 397 (1986).

8. A. P. Crawley and R. M. Henkelman, Magn. Reson. Med. 4, 34 (1987).

9. A. Fransson, A. Ericsson, and G. O. Sperber, Magn. Reson. Imaging 11, 197 (1993).

10. A. Fransson, A. Ericsson, and G. O. Sperber, Magn. Reson. Imaging 11, 1185 (1993).

11. J. Simbrunner and R. Stollberger, J. Magn. Reson. B 109, 301 (1995).

12. S. Majumdar, S. C. Orphanoudakis, A. Gmitro, M. O'Donnell, and J. C. Gore, Magn. Reson. Med. 3, 562 (1986).

13. A. Ross, M. Czisch, and G. C. King, J. Magn. Reson. 124, 355 (1997).

14. A. A. Maudsley, J. Magn. Reson. 69, 488 (1986).

15. A. J. Shaka, S. P. Rucker, and A. Pines, J. Magn. Reson. 77, 606 (1988).

16. T. Gullion, D. B. Baker, and M. S. Conradi, J . Magn. Reson. 89, 479 (1990).

17. M. Czisch, G. C. King, and A. Ross, J. Magn. Reson. 126, 154 (1997).

18. M. H. Levitt and R. Freeman, J. Magn. Reson. 43, 65 (1981).

19. A. Abragam, "Principles of Nuclear Magnetism," Clarendon Press, Oxford, 1961.

20. J. Baum, R. Tycko, and A. Pines, J. Chem. Phys. 79, 4643 (1983).

21. F. Hioe, Phys. Rev. A 30, 2100 (1984).

22. M. Silver, R. J oseph, and D. Hoult, J. Magn. Reson. 59, 347 (1984).

23. K. Uǧurbil, M. Garwood, A. R. Rath, and M. R. Bendall, J. Magn. Reson. 78, 472 (1988).

24. V. L. Ermakov, J .-M. Bohlen, and G. Bodenhausen, J. Magn. Reson. A 103, 226 (1993).

25. C. S. Poon and R. M. Henkelman, J. Magn. Reson. A 116, 161 (1995).

26. M. R. Bendall, M. Garwood, K. Uǧurbil, and D. Pegg, Magn. Reson. Med. 4, 493 (1987).

27. M. Garwood and Y. Ke, J. Magn. Reson. 94, 511 (1991).

28. S. Conolly, D. Nishimura, and A. Macovski, J. Magn. Reson. 83, 324 (1989).

29. T.-L. Hwang, P. C. M. van Zijl, and M. Garwood, J. Magn. Reson. 124, 250 (1997).

30. S. Conolly, G. Clover, D. Nishimura, and A. Macovski, Magn. Reson. Med. 18, 28 (1991).
31. M. H. Levitt and R. Freeman, J. Magn. Reson. 43, 502 (1981).

32. P. Fucini, C. Renner, C. Herberhold, A. A. Noegel, and T. A. Holak, Nature Struct. Biol. 4, 223 (1997).

33. F. Bloch, Phys. Rev. 70, 460 (1946).

34. R. R. Ernst, G. Bodenhausen, and A. Wokaun, "Principles of Nuclear Magnetism in One and Two Dimensions," Clarendon Press, Oxford, 1992.

35. J. Baum, R. Tycko, and A. Pines, Phys. Rev. A 32, 3435 (1985).

36. A. J. Shaka and J. Keeler, Prog. NMR Spectrosc. 19, 47 (1987).

37. M. H. Levitt, R. Freeman, and T. Frenkiel, J. Magn. Reson. 47, 328 (1982).

38. T. Fujiwara and K. Nagayama, J. Magn. Reson. 77, 53 (1988).

39. R. E. Walpole and R. H. Meyers, "Probability and Statistics for Engineers and Scientists," Macmillan, New York, 1978.

40. L. E. Kay, L. K. Nicholson, F. Delaglio, A. Bax, and D. A. Torchia, J . Magn. Reson. 97, 359 (1992).

41. A. Tannus and M. Garwood, J. Magn. Reson. A 120, 133 (1996).

42. S. R. Hartmann and E. L. Hahn, Phys. Rev. 128, 2042 (1962).

43. J. W. Peng, V. Thanabal, and G. Wagner, J. Magn. Reson. 94, 82 (1991).

44. J. W. Peng, V. Thanabal, and G. Wagner, J. Magn. Reson. 95, 421 (1991).

45. Bruker Analytische Messtechnik GmbH, "UXNMR," Rheinstetten, 1991.

46. C. Cieslar, A. Ross, T. Zink, and T. A. Holak, J. Magn. Reson. B 101, 97 (1993).

47. J . Callahan, D. Mattiello, and G. P. Drobny, Bull. Magn. Reson. 14, 191 (1993).

48. N. A. Farrow, R. Muhandiram, A. U. Singer, S. M. Pascal, C. M. Kay, G. Gish, S. E. Shoelson, T. Pawson, J. D. Forman-Kay, and L. E. Kay, Biochemistry 33, 5984 (1994).

49. J. W. Peng, and G. Wagner, Methods Enzymol. 239, 563 (1994).

50. N. J. Skelton, A. G. Palmer III, M. Akke, J. Kördel, M. Rance, and W. J. Chazin, J. Magn. Reson. B 102, 253 (1993).

51. U. Kamath and J. W. Shriver, J. Biol. Chem. 264, 5586 (1989).

52. A. G. Palmer III, M. Rance, and P. E. Wright, J. Am. Chem. Soc. 113, 4371 (1991).

53. V. Sklenář, M. Piotto, R. Leppik, and V. Saudek, J. Magn. Reson. A 102, 241 (1993).

54. A. J . Shaka, P. B. Barker, and R. Freeman, J . Magn. Reson. 64, 547 (1985).

55. D. Marion, M. Ikura, R. Tschudin, and A. Bax, J. Magn. Reson. 85, 393 (1989).

56. A. G. Palmer III, N. J . Skelton, W. J . Chazin, P. E. Wright, and M. Rance, Mol. Phys. 75, 699 (1992). 